\title{
The Japanese Community Pharmacists' Perceptions of the Health Support Pharmacy System
}

\author{
Noritake Hirota ${ }^{1,2 *}$, Noboru Okamura1 \\ ${ }^{1}$ Department of Clinical Pharmacy, Faculty of Pharmaceutical Sciences, Mukogawa Women's University, Nishinomiya, Japan \\ ${ }^{2}$ General Incorporated Association Osaka Pharmaplan, Aozora Pharmacy, Osaka, Japan \\ Email: *n-hirota@faruma.co.jp
}

How to cite this paper: Hirota, N. and Okamura, N. (2020) The Japanese Community Pharmacists' Perceptions of the Health Support Pharmacy System. Pharmacology \& Pharmacy, 11, 55-70.

https://doi.org/10.4236/pp.2020.114006

Received: March 15, 2020

Accepted: April 27, 2020

Published: April 30, 2020

Copyright (c) 2020 by author(s) and Scientific Research Publishing Inc. This work is licensed under the Creative Commons Attribution International License (CC BY 4.0).

http://creativecommons.org/licenses/by/4.0/

\begin{abstract}
A questionnaire survey was conducted employing pharmacists at 350 Japanese Federation of Democratic Medical Institutions (Min-Iren) member pharmacies, evaluating the challenges related to registering as a Health Support Pharmacy (HSP). Completed responses were received from 193 pharmacies (55.1\%). Fifty-five (28.5\%) of these pharmacies were approved as meeting the HSP criteria. Some difficulties encountered in registering as an HSP included the preparation of the numerous required documents and placing a health support pharmacist. The obstacles that prevented pharmacists from registering were physical, including "placement of two or more health support pharmacists" and "placement of the required over-the-counter drugs (OTCs)." Based on these, $51.8 \%$ of supervising pharmacists stated the opinion that the government should "loosen current criteria." However, a survey conducted among the 724 participant pharmacists, working in a pharmacy, showed no significant difference in their answering tendencies depending on whether or not they worked at an HSP. This could be attributed to the effort that MinIren pharmacies have been putting into health support and primary care functions. Overall, $64.5 \%$ of pharmacists working at HSPs were proud that the pharmacy they worked had HSP status. The present survey revealed the physical obstacles of HSP registration. Conformity to the criteria of HSP is essential for performing their fundamental functions as pharmacies. The relaxation of the criteria is anticipated.
\end{abstract}

\section{Keywords}

Health Support Pharmacy, Health Support Function, Primary Care Function 


\section{Introduction}

In Japan, the history of pharmacies and pharmacists is extremely recent compared to European countries. The European system of pharmacies was learned and modeled as Japan entered the Meiji period; that is, the beginning of its history as a modern nation [1]. A prominent fundamental difference between the Japanese and European healthcare system lies in the authority of physicians who can dispense their own prescription drugs. Until the establishment of the national health insurance system in 1961, financial constraints restricted the availability of healthcare to all citizens. This was particularly true for independent business owners and workers in the agriculture and fisheries sectors, who were previously not covered by health insurance. Therefore, it is not an understatement that pharmacy sales of pharmaceutical products were the foundation of the national healthcare system, as well as one of the fundamental roles of pharmacies/pharmacists in assisting in what would be referred to as "self-medication" in modern terms. However, with the recent rapid increase in prescription drugs, the number of so-called traditional pharmacies has decreased. This resulted in the emergence of pharmacies specialized in prescription drugs that do not sell any over-the-counter (OTC) drugs. In other words, the roles of pharmacies and pharmacists have changed through the movement to separate medical practice and drug dispensation. However, this is now recognized as a problem by both citizens and the medical community. The Ministry of Health, Labor and Welfare (MHLW) has taken the initiative to discuss the future of pharmacies/pharmacists in the era of Community-based Integrated Care that will be enacted in 2025. In October 2015, the MHLW published the "Pharmacy Vision for Patients" (Figure 1) [2].

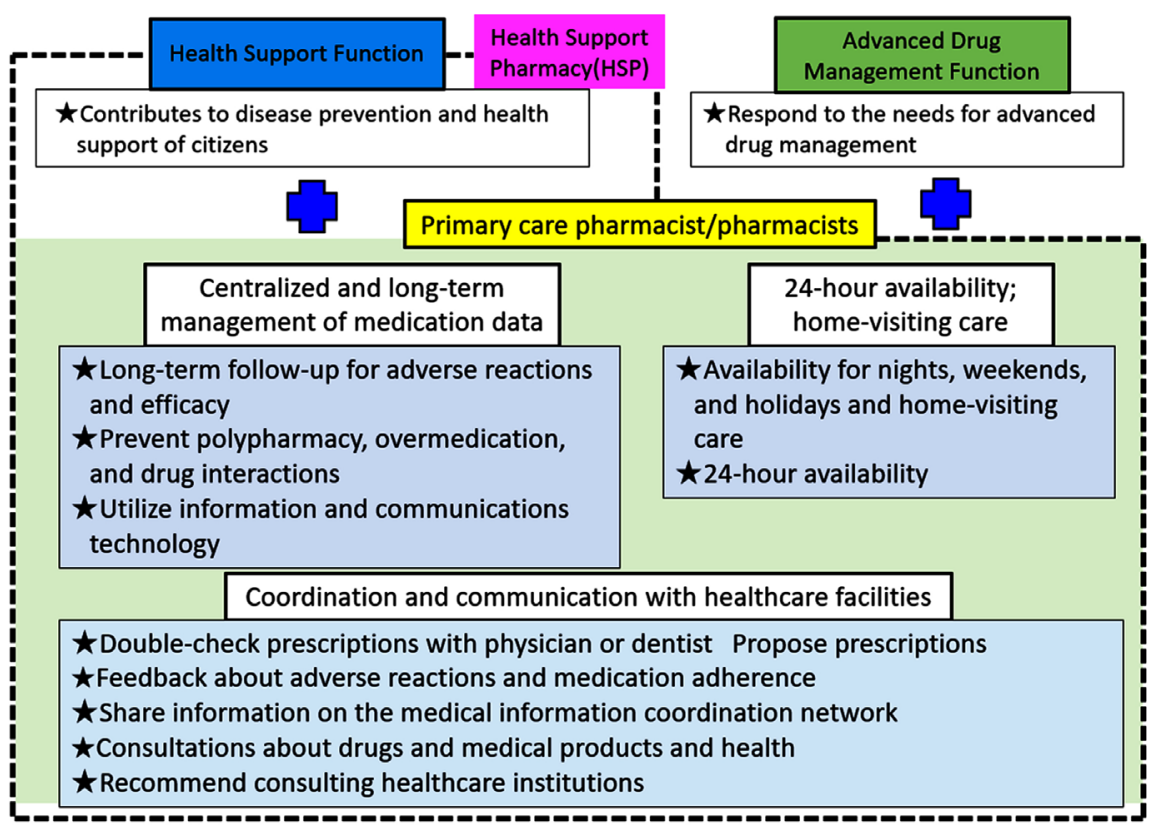

Figure 1. Pharmacy Vison for patients. 
Under the "Pharmacy Vision for Patients," all pharmacies and pharmacists are expected to serve the following two broad functions by 2025: 1) "primary care function"; i.e., centralized management of prescriptions, home services, 24-hour availability, coordination with medical, and long-term care facilities, and 2) "health support function" which aims to promote health in community-dwelling residents, preventive medicine, and self-medication, as well as coordinate services with Advanced and Specialized Medical Care Institutions that prescribe anticancer and HIV drugs. The HSP system was newly established in October 2016 to institutionalize HSP that embodies both the "health support" and "primary care" functions of pharmacies. As the goal for the 2025 inauguration of the Community-based Integrated Care system, the MHLW has announced a target of at least one HSP operating within each middle school district (equivalent to approximately 15,000 pharmacies nationwide). Unfortunately, as of in December 2019, there were only approximately 1800 pharmacies, which is only $12 \%$ of the target, accounting for approximately $3 \%$ of all pharmacies. Three years have passed since the launch of the HSP system, but the movement has remained slow. There are several arguments concerning the lack of progress, with no thorough studies on this topic to date.

Thus, we conducted a questionnaire survey among supervising and employed pharmacists of member pharmacies of the Japanese Federation of Democratic Medical Institutions (Min-Iren) to explore the factors preventing pharmacies from registering as an HSP.

\section{Methods}

\subsection{Participants}

Participants were Min-Iren member pharmacies. As of June 2019, there were 350 member pharmacies of the Min-Iren.

\subsection{Survey Methods and Period}

Questionnaire forms for the supervising pharmacists and for employed pharmacists were sent to the pharmacies in June 2019; completed questionnaires were mailed back in the enclosed return envelopes. The survey period was July-October 2019. The questionnaire forms to be answered by supervising pharmacists were not anonymous, while the questionnaires for employed pharmacists were anonymous. The survey evaluating full-time pharmacists was only conducted among permanent employees. Part-time and dispatch pharmacists were excluded.

\subsection{Survey Items}

\subsubsection{Questionnaire for Supervising Pharmacists}

1) Name, sex, age; 2) Basic dispensing fee at the pharmacy; 3) whether or not the pharmacy is registered for community pharmacy service premiums (chiiki shien taisei kasan); 4) whether it is registered for generic substitution premiums (kouhatsu iyakuhin taisei kasan); 5) number of pharmacists who have completed the 
health support pharmacist training; 6) HSP registration status; 7) challenges faced in the HSP registration; 8) exemplary moments experienced in the HSP; 9) intention/interest in registering pharmacies currently not registered as HSP; 10) reasons for not registering as HSP; 11) criteria missing for HSP registration; 12) reasons for "having no intention to register" or "choosing not to register despite meeting criteria"; 13) expectations towards the MHLW or the local government (pharmaceutical division) related to HSP; and 14) expectations towards the Japanese Pharmaceutical Association or local pharmacists' association in regards to HSP.

\subsubsection{Questionnaire for Employed Pharmacists}

1) Age and sex/years of experience as a community pharmacist/job title within the pharmacy; 2) "primary care pharmacist" facility criteria and number of patients; 3) perceptions on the "primary care pharmacist" initiative and details; 4) reasons for not acquiring the "primary care pharmacist" facility criteria; 5) awareness that the pharmacy they are working in was an HSP; 6) how pharmacists who worked at an HSP assessed the system; 7) reason why the pharmacy they worked in is not an HSP; 8) experience in being consulted regularly regarding drugs and medical products or health information by patients and community residents outside of home-visiting care or guidance related to medication; if so, the frequency.

\subsection{Methods of Data Aggregation and Analysis}

The contents of the collected questionnaires were entered into Microsoft Excel 2010 to enumerate the respondents. A test of independence was performed to ascertain whether the number of respondents between HSP and other pharmacies differed. KH Coder 3 was used for text mining analysis of free-response items [3].

\subsection{Statistical Analysis}

Independence of factors was tested by the Chi-square test using EZR ver.1.27 (Jichi Medical University Saitama Medical Center) [4]. A risk rate with $p<0.05$ was considered statistically significant.

\subsection{Ethical Considerations}

The study was conducted in compliance with the "Declaration of Helsinki Ethical Principles for Medical Research Involving Human Subjects" and was approved by the Ethics Committee of Mukogawa Women's University (Approval No. 19-01) and the Ethics Committee of the Japanese Federation of Democratic Medical Institutions (Min-Iren) (Approval No. 43-004).

\section{Results}

\subsection{Collection Rate}

To date, questionnaire forms were sent to 350 Min-Iren member pharmacies 
(June 2019). By October 31, 2019, responses were received from 193 pharmacies (55.1\%). Questionnaires for the survey of supervising pharmacists were distributed to 193 respondents; i.e., the same number as the number of cooperating pharmacies, and questionnaires for the survey of employed pharmacists were distributed to 745 pharmacists at cooperating pharmacies. Responses were obtained from 724 pharmacists ( $97.2 \%$ collection rate).

\subsection{Results of the Survey for Supervising Pharmacists}

\subsubsection{The Facility Criteria of Cooperating Pharmacies and Age and Sex of Supervising Pharmacists}

At the cooperating pharmacies, the category for basic fees on prescription receipt was " 1 " in 143 pharmacies (74.1\%), " 2 " in 44 pharmacies (22.8\%), "Other" in 4 pharmacies $(2.1 \%)$, and 2 pharmacies left this response blank (1.0\%) (Table 1 (a)). With regards to registering for community pharmacy service premiums, 125 pharmacies (64.8\%) answered that were registered, 67 pharmacies answered that they were not (34.7\%), and 1 pharmacy left this question blank (0.5\%) (Table 1(b)). With regards to the generic substitution premium category, 20 pharmacies (10.4\%) were category " 1 " ( $\geq 75 \%$ of dispensed drugs were generics), 45 pharmacies $(23.3 \%)$ were " 2 " ( $\geq 80 \%$ of dispensed drugs were generics), and 125 pharmacies $(64.8 \%)$ were " 3 " ( $\geq 85 \%$ of dispensed drugs were generics). Three pharmacies (1.5\%) left this question blank or answered that its factor was "not calculated" (Table 1(c)).

Table 1. Facility criteria of pharmacies that participated in the survey. (a) Basic dispensing fee category; (b) Registration for community pharmacy service premiums; (c) Registered generic substitution premium category.

(a)

\begin{tabular}{cc}
\hline 1 & $143(74.1 \%)$ \\
2 & $44(22.8 \%)$ \\
Other & $4(2.1 \%)$ \\
Blank response & $2(1.0 \%)$ \\
Total & $193(100.0 \%)$ \\
\hline
\end{tabular}

(b)

\begin{tabular}{cc}
\hline Registered & $125(64.8 \%)$ \\
Not registered & $67(34.7 \%)$ \\
Blank response & $1(0.5 \%)$ \\
Total & $193(100.0 \%)$ \\
\hline & $(\mathrm{c})$ \\
\hline $1(75 \% \geq)$ & $20(10.4 \%)$ \\
$3(80 \% \geq)$ & $45(23.3 \%)$ \\
Blank response or not calculated & $125(64.8 \%)$ \\
Total & $3(1.5 \%)$ \\
\hline
\end{tabular}


A mean of 1.7 pharmacists per pharmacy had completed training as a health support pharmacist ( $\min =0$ pharmacists in 46 pharmacies; $\max =11$ pharmacists in 1 pharmacy). In case of HSPs, a mean of 3.1 (range 2 - 11) pharmacists had completed this training. Overall, 138 pharmacies reported one or more health support pharmacists (71.5\%) and 55 pharmacies had none (28.5\%).

Seventy-eight of the supervising pharmacists were male (40.4\%) and 115 were female (59.6\%). The mean age of supervising pharmacists was 47.3 (range: 30 $67)$ years.

\subsubsection{Registration Status as Health Support Pharmacy}

Fifty-five pharmacies (28.5\%), or $100 \%$ of Min-Iren HSP, were registered as HSP. A total of 124 (64.2\%) were not registered and 14 pharmacies (7.3\%) were "preparing for registration."

\subsubsection{Difficulties Faced in Health Support Pharmacy Registration (Multiple Selections Allowed)}

The most common response was "too many documents to prepare" (49 pharmacies), followed by "securing a pharmacist who has completed health support pharmacist training" (48 pharmacies), "stocking OTC and medical goods" (28 pharmacies), "holding events such as health classes" (28 pharmacies), "strict instructions of the government office representative (pharmaceutical division)" (5 pharmacies), and "Other" (3 pharmacies).

\subsubsection{Intention to Register as a Health Support Pharmacy for Currently Unregistered Pharmacies}

Fifty-five pharmacies (39.6\%) answered "Yes" and 29 (21.9\%) answered "No", while 50 pharmacies (36.0\%) were "still investigating", and $5(3.6 \%)$ left the response unanswered.

\subsubsection{Reasons for Not Registering as Health Support Pharmacy}

One reason for not registering was indicated as "not meeting criteria for registration," a reply provided by 55 pharmacies (39.6\%). Here, the most common reason (multiple selections allowed) was "inability to place the required OTC on the shelves" (74 pharmacies), followed by "not having two or more pharmacists who have completed health support pharmacist training" (73 pharmacies), "inability to hold events such as health classes" (45 pharmacies), "lack of space with privacy" (31 pharmacies), "business hours that do not meet the criteria" (12 pharmacies), "lack of home visiting services" (5 pharmacies), and "lack of primary care pharmacists" (5 pharmacies).

The second reason for not applying for registration was "choosing not to register despite meeting criteria," which was true in the case of 29 pharmacies (21.0\%), followed by "Other" for 50 pharmacies (36.2\%). Five pharmacies (3.6\%) left this question unanswered.

\subsubsection{Reasons for Choosing Not to Register Despite Meeting Criteria (Multiple Selections Allowed)}

Six pharmacies answered that "they already function as HSP without necessarily 
registering that status" (6 pharmacies), with four reasoning that "there are no benefits in terms of dispensing fees" (4 pharmacies), while others implied reasons such as "cumbersome preparation of the documents required for registration" (4 pharmacies), "lack of consensus in the workplace" (3 pharmacies), and "Other" (27 pharmacies).

\subsubsection{Expectations in the MHLW and the Local Government (Pharmaceutical Division) Related to the Health Support Pharmacy System}

While four pharmacies believed that "current criteria were not strict enough" (2.1\%), 99 pharmacies believed that the "current criteria should be loosened" (51.6\%), 79 pharmacies (41.1\%) responded "Nothing in particular," and 10 pharmacies (5.2\%) left this unanswered.

\subsubsection{Analysis of Free Responses}

$\mathrm{KH}$ Coder3 was used for text mining the 259 free responses related to the respondent opinions on HSP registration, and opinions related to the local government (pharmaceutical division), the Japanese Pharmaceutical Association and local pharmacists' association. Several opinions were related to the health support pharmacist training and inventory of OTC drugs (Figure 2).

\subsection{Results of the Survey for Employed Pharmacists}

Of the 724 full-time pharmacists who replied, 301 worked at HSPs (41.6\%). For the following listed items, the answers of HSP pharmacists were analyzed separately from those of other pharmacists.

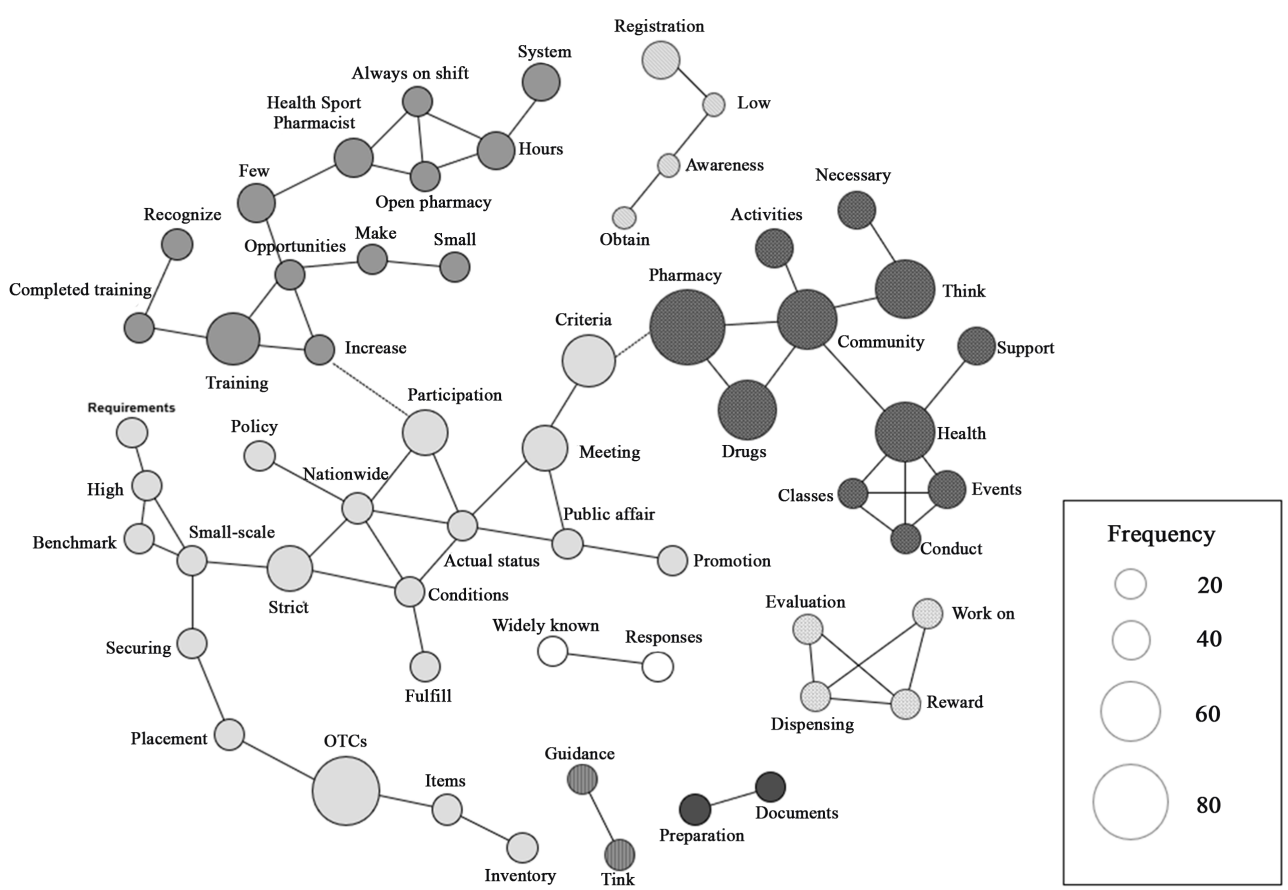

Figure 2. Co-occurrence network analysis of free responses. Translations of bubbles, generally from left to right, top to bottom. 
3.3.1. Age Composition, Sex, and Years of Experience as a Pharmacist No significant differences were observed between the two groups in terms of age composition $(p=0.325)$ (Figure 3(a)), sex $(p=0.203)$ (Figure $3(b))$ or years of experience as a pharmacist $(p=0.203)$ (Figure $3(c))$.

\subsubsection{Roles within the Pharmacy}

Though the ratio of supervising pharmacists was significantly lower in the HSP $(p=0.003)$, no significant differences were observed in the numbers of pharmacists with other job titles (Figure 4).

\subsubsection{Registration Status of Primary Care Pharmacist Facility}

Overall, 408 pharmacists (54.6\%) were registered for the facility status of "primary care pharmacist"; no significance was observed between the two groups ( $p$ $=0.224$ ) (Figure 5). At pharmacies that meet the facility criteria of "primary care pharmacist", the pharmacist functioned as the "primary care pharmacist" at a pharmacist-to-patient ration of 1:8.4 \pm 11.9 . No significant differences were observed between the ratio of pharmacists who work in HSP (1:7.6 \pm 9.3$)$ and those who work in other pharmacies $(1: 8.9 \pm 14.2)(p=0.782)$.

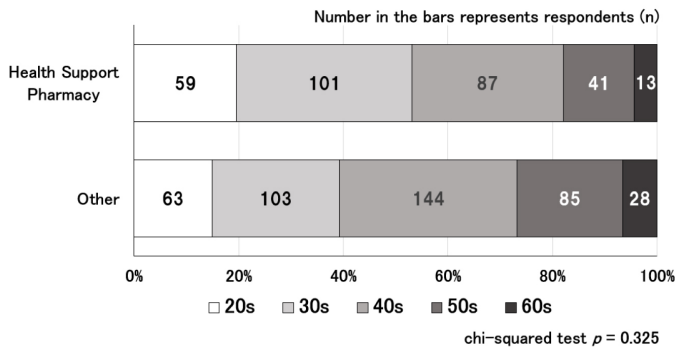

(a)

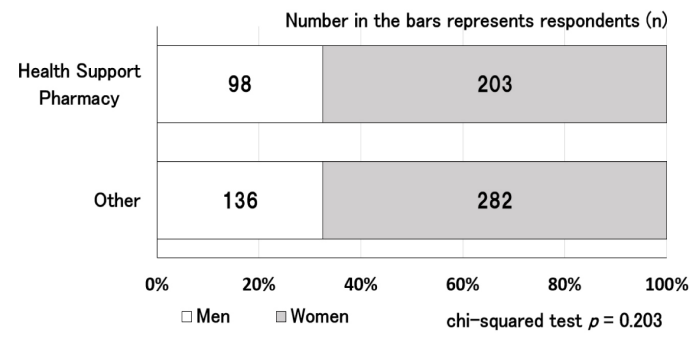

(b)

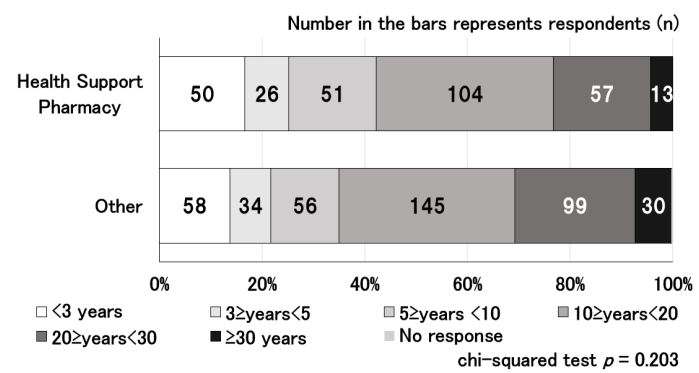

(c)

Figure 3. Age composition, sex, years of experience as a pharmacist. (a) Age distribution; (b) Sex; (c) Years of experience as a pharmacist in a community pharmacy. 


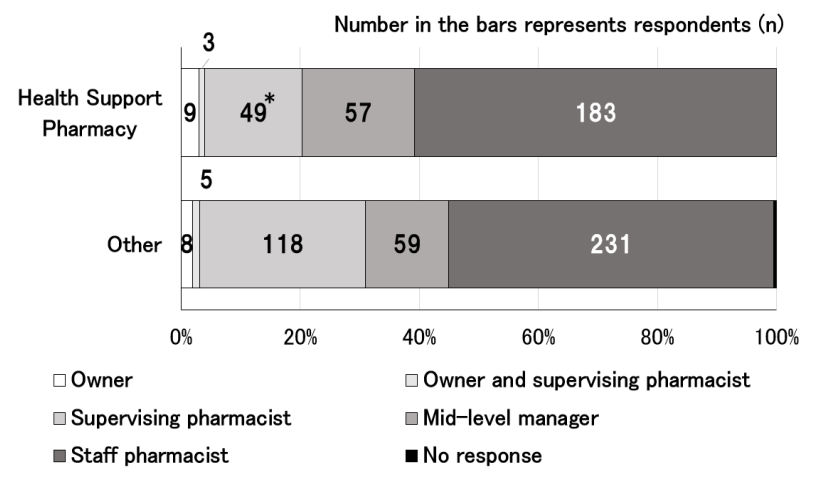

Figure 4. Job title within the pharmacy Chi-squared test *: The rate of "HSP" was significantly higher than “Other" $(p=0.003)$.

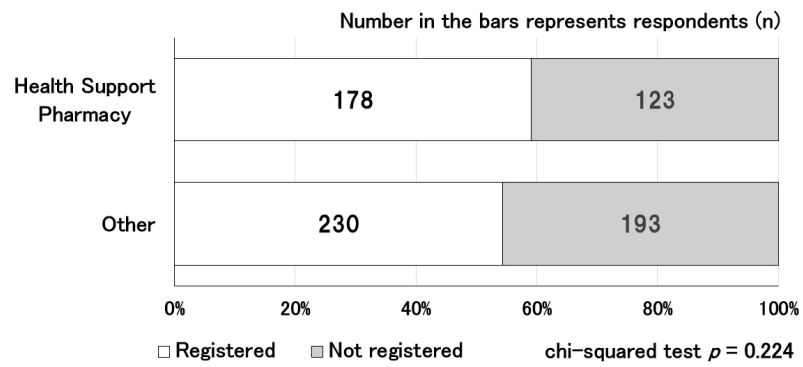

Figure 5. Facility registration status as "primary care pharmacist".

\subsubsection{Assessment of "Primary Care Pharmacist" Activities}

Overall, 233 pharmacists (57.1\%) assessed the activities of the "primary care pharmacist" system positively, with 123 pharmacists (30.1\%) answering "Neither". This suggested the presence of some disparities in their opinions of the system. However, there only a small number of pharmacists assessed it negatively (17 pharmacists, $4.3 \%)$. No significant difference was observed between the two groups for this assessment $(p=0.593)$ (Figure 6).

The most commonly mentioned benefit of the "primary care pharmacist" system allowed pharmacists to "gain the trust of patients under their charge" (187 pharmacists), followed by "maximize their professional utility as pharmacist" (108 pharmacists), "manage patients under their care with all relevant data on prescriptions and OTCs" (98 pharmacists), "follow up on patients under their care for 24 hours a day 365 days a year" (Table 2(a)).

Negative aspects of the "primary care pharmacist" system included "being bound 24 hours a day 365 days a year" (7 pharmacists), "need to individually treat each patient" (4 pharmacists), and "extra time taken to dispense drugs and educate patients on their medications" (1 pharmacist) (Table 2(b)).

\subsubsection{Reasons for Not Registering the Facility as "Primary Care Pharmacist"}

We questioned 316 pharmacists regarding the reasons for not meeting the "primary care pharmacist" facility criteria. Multiple selections were allowed. A total of 250 pharmacists answered that "the facility criteria were not met," 19 replied 


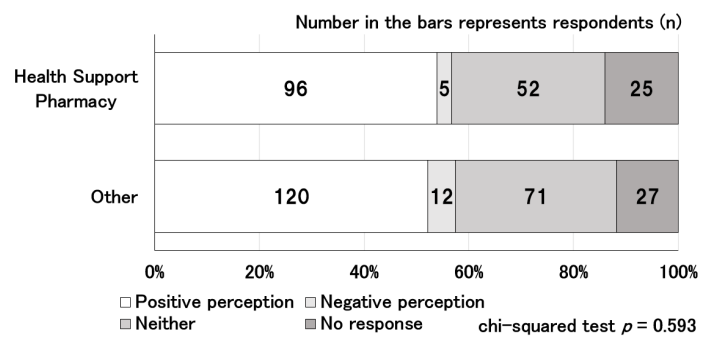

Figure 6. Perceptions on the "primary care pharmacist" initiative.

Table 2. Assessment of the "primary care pharmacist" system. (a) Positive aspects; (b) Negative aspects.

(a)

\begin{tabular}{cccc}
\hline The "primary care pharmacist" system allows me to & HSP & Other & Total \\
\hline $\begin{array}{c}\text { Gain the trust of patients under their charge } \\
\text { Maximize my professional utility as pharmacist }\end{array}$ & 87 & 100 & 187 \\
Manage patients under my care with & 48 & 60 & 108 \\
all relevant data on prescriptions and OTCs & 41 & 57 & 98 \\
Follow up on patients under my care 24 hours a day 365 days a year & 9 & 12 & 21 \\
Other & 9 & 12 & 21 \\
\hline
\end{tabular}

${ }^{\star}$ multiple selections allowed.

(b)

\begin{tabular}{cccc}
\hline The "primary care pharmacist" system allows me to & HSP & Other & Total \\
\hline $\begin{array}{c}\text { Being bound for } 24 \text { hours a day } 365 \text { days a year } \\
\text { Need to treat differentially from other patients }\end{array}$ & 2 & 5 & 7 \\
Extra time taken to dispense drugs and & 1 & 3 & 4 \\
educate patients on their medications & 0 & 1 & 1 \\
Other & 2 & 6 & 8
\end{tabular}

*multiple selections allowed.

"they were personally not interested in doing this proactively," 14 said it was according to the "pharmacy policy which did not designate them to become "primary care pharmacists," 17 answered "Other", and 30 failed to respond.

In the case of the facility criteria, among the pharmacists who met the minimum condition of " 3 or more years of experience in a pharmacy," the reasons for "Other" (multiple selections allowed) were "not being a certified pharmacist " (63 pharmacists), "not having worked at the pharmacy for one or more years," (52 pharmacists), "not participating in community activities related to healthcare" (43 pharmacists), and "work hours do not match the designated hours" (13 pharmacists).

\subsubsection{Awareness on Whether or Not Their Pharmacy Was Registered as Meeting "Health Support Pharmacy" Criteria}

Overall, $94.7 \%$ of pharmacists working at HSPs (285 pharmacists) were aware of their pharmacy's designation. However, 11 (3.6\%) answered that they were not 
aware or were unsure of the status. Furthermore, $6.3 \%$ of pharmacists working at non-HSP (26 pharmacists) falsely assumed that their pharmacy had HSP status (Figure 7).

The most common reason for replying "not registered" (multiple selections allowed) were "not meeting criteria despite wanting to register as one" (238 pharmacists), followed by "to avoid increased workload after becoming an HSP" (42 pharmacists), "they already function as an HSP without necessarily registering for that status" (21 pharmacists), and "not in line with company or pharmacy policy" (16 pharmacists), indicating that many pharmacies were interested in registering for HSP status, but failed to meet all criteria.

\subsubsection{Pride That the Pharmacy Is a "Health Support Pharmacy"}

In total, 285 pharmacists working at HSPs and aware of the status answered that they strongly agreed with the statement, "I am proud that my pharmacy is an HSP (48 pharmacists, 16.8\%), 136 answered that they agreed (47.7\%), 85 answered neither (29.8\%), 7 answered that they disagreed (2.5\%), 1 answered that he strongly disagreed $(0.4 \%)$, with 8 leaving the question unanswered $(2.8 \%)$. Overall, 64.5\% were proud of their pharmacy's HSP designation (Figure 8(a)). Pharmacists recognized the HSP designation most distinctly through activities such as "providing information to pharmacy users and community residents in health classes" (188 pharmacists, 66.0\%), followed by "health check activities such as measuring blood pressure or body fat in and out of the pharmacy" (45 pharmacists, $15.8 \%$ ), and "sales of OTC and supplements and consultation activities" (30 pharmacists, 10.5\%) (Figure 8(b)).

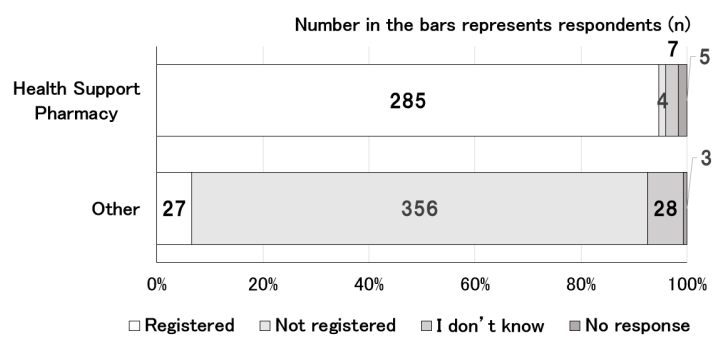

Figure 7. Knowledge of whether the pharmacy was registered "HSP".

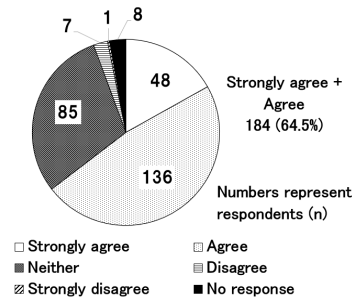

(a)

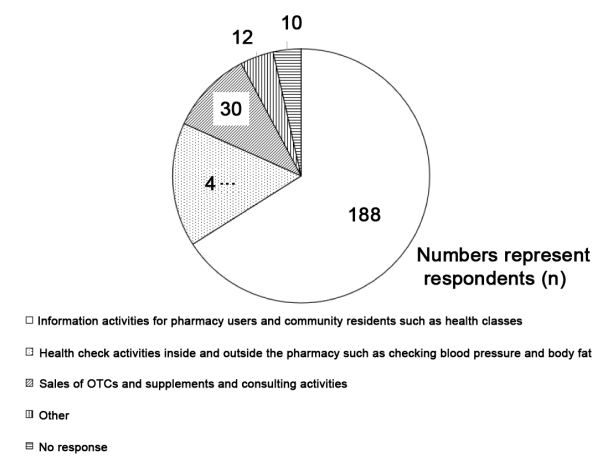

(b)

Figure 8. Pride in working at "HSP”. 


\subsubsection{Experience and Frequency of Responding to Patient or Community Resident Consultation Requests Related to Drugs and Health (In-Person or Phone), Outside of Home Visiting Services or Medication-Related Guidance at the Pharmacy}

In total, 617 pharmacists (85.2\%) answered that they possessed experience (Figure 9(a)) at a frequency of "About once a week" in 29.8\%, "About once a month" in 30.6\%, "About once a year" in 5.0\%, and "Frequency unknown" in $34.0 \%$. No response was provided by $0.5 \%$ of respondents. With regard to this, pharmacists working at an HSP replied that the "Frequency of receiving an unknown question" was significantly lower $(p=0.046)$ (Figure 9(b)).

\section{Discussion}

In the present study, we conducted a questionnaire survey among Min-Iren member pharmacies to analyze the factors underlying the low registration rates of HSP based on the pharmacists' perspective. Furthermore, the status of registration as "primary care pharmacist" and their assessments of the system, and opinions on HSP were sought in the survey for conducted among full-time pharmacists employed at pharmacies.

\subsection{Pharmacy Size and Facility Criteria}

The pharmacy size can be inferred from the basic dispensing fee category. Basic dispensing fee 1 is the category for pharmacies with less than $85 \%$ of prescriptions from a specific health care institution and with less than 2000 prescription receipts per month. According to MHLW statistics, pharmacies in this category comprise $76.9 \%$ of all pharmacies, while $7.9 \%$ are categorized as basic dispensing fee 2 and $15.4 \%$ as "Other" (June 2018 assessment) [5]. Compared to these figures, $74.1 \%$ of pharmacies in the present survey were categorized as " 1 ", $22.8 \%$ as " 2 ", and $2.1 \%$ as "Other"; herein, the percentage of pharmacies categorized as " 2 " was higher than the national rate. This is presumably because a higher proportion of Min-Iren pharmacies received prescriptions from hospitals (pharmacies with high prescription demand).

Next, with regard to the facility criteria for community pharmacy service premiums, 15,382 pharmacies earned community pharmacy service premiums

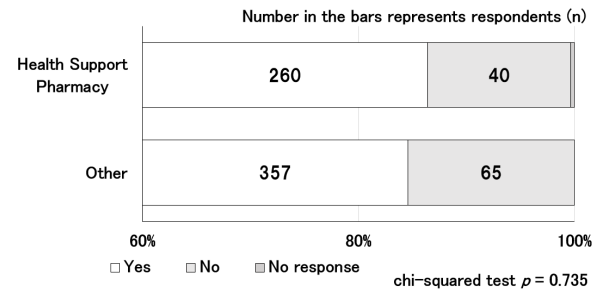

(a)

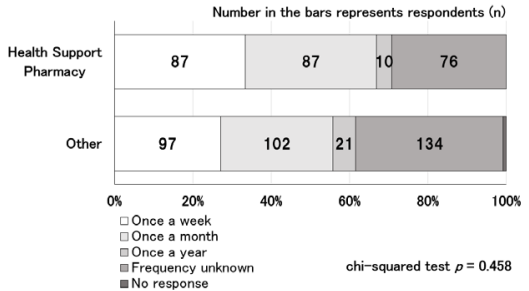

(b)

Figure 9. Experiences and frequency of responding to patient or community resident consultation requests related to drugs and health, outside of home visiting services or medication-related guidance at the pharmacy. (a) Experience of being consulted; (b) Frequency of being consulted. 
as of July 1,2018 , amounting to $26.7 \%$ of pharmacies calculating basic dispensing fees. In the present survey, $64.8 \%$ of surveyed Min-Iren pharmacies calculated basic dispensing fees. Community pharmacy service premiums require 1) prescription of narcotics; 2) home visiting services; 3) registration of primary care pharmacists; 4) $\geq 45$ hours of business hours per week; 5) drug and medical supply inventory $\geq 1200$ items; 6) 24 -hour on-call availability; 7) $\geq 50 \%$ proportion of generics dispensed; 8) PMDA Navigator registration; 9) reporting on prevented and avoided adverse drug reaction cases and 10) established system to report adverse reactions. Pharmacies that calculate their fees under the basic dispensing fee category 1 are required to meet even more stringent requirements and the community pharmacy service premium is a way to reward pharmacies functioning as "primary care pharmacies/pharmacists" as described in the "Pharmacy Vision for Patients" (Figure 1) through higher dispensing fees. Based on these findings, the surveyed Min-Iren pharmacies in this study served their primary care roles more than the national average.

Next, Min-Iren pharmacies and other pharmacies nationwide (as of the present, July 1, 2019) were compared in terms of generic substitution premiums, resulting in the "Premium Category 1" national average: 36.5\%, Min-Iren: 10.4\%, "Premium Category 2" national average: 34.4\%, Min-Iren: 23.3\%, "Premium Category 3" national average: $29.1 \%$, Min-Iren: 64.8\%, demonstrating that Min-Iren pharmacies played important roles as promoters of generic drug use. This may be explained by the effort and emphasis placed by Min-Iren healthcare facilities and pharmacies in optimizing medical costs and reducing out-of-pocket fees.

\subsection{Health Support Pharmacy Registration}

Next, we investigated the HSP registration status. As of June 2019, there were 350 Min-Iren member pharmacies, of which 55 were HSP (15.7\%). Considering that there were 1567 health support pharmacists (2.7\%) nationwide according to the MHLW report in September 2019, Min-Iren pharmacies are active promoters of the HSP system. In addition, in the current study, the HSP response rate was $100 \%$ compared to other pharmacies (46.8\%), indicating that supervising HSP pharmacists were positive in their cooperation of the present survey.

The training of health support pharmacists started with the inauguration of the HSP system. According to the "Survey Report on Primary care Pharmacists and Pharmacies" published by the MHLW in March 2019 [6], an average of 0.5 pharmacists had completed the health support pharmacist training per pharmacy. In comparison, an average of 1.7 pharmacists had completed the training in Min-Iren pharmacies, which was over three times the national average. This figure further reflects that Min-Iren pharmacies are active promoters of the HSP system.

Collectively, it appears that the two main challenges faced by supervising pharmacists of Min-Iren HSP in the status registration were "too many documents to prepare" and "securing a pharmacist who had completed health sup- 
port pharmacist training." The current criteria of HSP requires a health support pharmacist to be present on shift at all times during business hours [7]. The same notification requires the average pharmacy's business hours to be 8 or more hours on weekdays and 4 or more hours on weekends, requiring them to be open for a minimum of 44 hours per week. Pharmacists working at pharmacies operated by companies are considered employees and are protected by the Labor Standards Law; thus, their weekly working hours would be 40 hours. Furthermore, the pharmacists are also ensured days off work. Thus, with the exception of independently operated pharmacies, it is impossible to maintain an HSP for all business hours with one health support pharmacist, while still conforming to all laws related to business hours. Thus, this necessitates the presence of at least two health support pharmacists per pharmacy. Additionally, the present survey revealed that an average of 3.1 pharmacists completed training in HSP, active efforts being made to place at least two per pharmacy in Min-Iren pharmacies.

According to the survey conducted by the MHLW [6], 38.4\% of pharmacies not meeting HSP criteria still intended to register. In the present survey, 69 such pharmacies, that were Min-Iren members (35.8\%), planned to register as HSP (some answered that they "have the intention to register" and were "Preparing for registration"), which was approximately equivalent to the survey results of the MHLW.

In regards to why they had not yet registered as HSP, the most common reason was "Not meeting the various criteria" (39.6\%), which was further elaborated by specific reasons of (difficulty in) "placing two health support pharmacists," "placing all the required OTC on the shelves," "inability to hold events such as health classes", and "lack of space with privacy", all of which related to physical conditions. Conditions related to the primary occupational tasks of pharmacies and pharmacists, such as "lack of home visiting services" or "lack of primary care pharmacists", did not hinder registration. Simultaneously, 29 pharmacies $(20.9 \%)$ replied that they were "choosing not to register despite meeting criteria", rationalizing that "they already functioned as an HSP," "preparation of the documents required for registration was cumbersome," and "there were no benefits in terms of dispensing fees", justifying that the benefits of registering as an HSP would not outweigh the tremendous effort needed to meet criteria. Herein, the respondents' expectations from the government were consistent, with $51.8 \%$ of the sample answering that the "current criteria should be loosened." In addition, the co-occurrence analysis from text mining results uncovered content related to the necessary numbers of health support pharmacists and OTC items (Figure 2).

Thus, for further promoting registration as HSP, first and foremost, the current criteria requiring a health support pharmacist at all business hour shifts need to be loosened to requiring just one per pharmacy. This is expected to promote registration by smaller pharmacies as well. 


\subsection{Perceptions of Employed Pharmacists on HSP}

Next, in the survey of employed pharmacists, no statistical significance was observed between pharmacists who worked at HSP versus other pharmacies. This is probably a reflection of how Min-Iren pharmacies have been valuing their roles as "primary care pharmacies" even before the inauguration of HSPs, engaging in health-promoting activities for pharmacy users and community residents [8]. On average, there were 8.4 patients per pharmacist who had signed the "Primary Care Pharmacist Consent Form." According to the MHLW survey [7], the average was 1.7 patients, which revealed the Min-Iren pharmacies' emphasis on their "primary care functions." Overall, $52.9 \%$ of pharmacists positively assessed the "primary care pharmacist" initiatives, with only $4.2 \%$ reporting a negative perception; thus, in general, the initiatives were supported (Figure 6). For example, this allowed them to "gain the trust of patients under their charge" and "manage patients under their care with all relevant data on prescriptions and OTCs." However, the similarities ended there as opinions were divided with regards to whether the "primary care function" should be undertaken by individual pharmacists or by the pharmacies. Furthermore, the MHLW is not explicit about this distinction, using expressions such as "primary care pharmacist/pharmacy" to include both. Additionally, it is important for pharmacists and patients to build one-on-one relationships; however, it is assumed that HSPs have centralized patient data management to serve the primary care function, regardless of whether a "Primary Care Pharmacist Consent Form" has been signed. Of the pharmacists working in a non-HSP $(n=27), 6.4 \%$ believed that the pharmacy they were working at was an HSP (Figure 8). As mentioned earlier, given that there were $20.9 \%$ of pharmacies (29 pharmacies) "choosing not to register despite meeting criteria," it was expected that some pharmacists falsely believed that the non-registered pharmacy they were working at was a registered HSP.

Furthermore, $64.5 \%$ of pharmacists working at an HSP reported a feeling of pride that their pharmacy was a registered HSP (Figure 8). A total of $85.2 \%$ pharmacists provided consultation to patients and community residents regarding medications and medical products in addition to the dispensing of prescription drugs and home visiting services. Among these pharmacists, $60.4 \%$ provided consultations at least once a month (Figure 9), demonstrating that the designation of HSP is founded on the practice to fulfill "health support" and "primary care" functions, and probably boosting the pharmacists' motivation.

\section{Conclusion}

The present study revealed the reasons underlying the lagging progress in the HSP system despite three years since its inauguration. These included physical obstacles, such as the number of health support pharmacists required and the placing of OTC on shelves, which imposed challenges even in Min-Iren pharmacies with comparatively high levels of registration. Min-Iren member HSPs 
served both the "primary care" and "health support" functions adequately, seemingly reflected by the feeling of the pride experienced by pharmacists working at these pharmacies. The activities of health support pharmacists are important for further enhancing the functions of pharmacies and the skills and motivation of pharmacists; however, the actualization of these improvements would rely on the effort and ingenuity of governmental bodies.

\section{Acknowledgements}

We would like to express our gratitude to the Board of Pharmacists of the Japanese Federation of Democratic Medical Institutions and the managers and pharmacists of the pharmacies that cooperated in our survey.

\section{Conflicts of Interest}

The authors declare no conflict of interest regarding the publication of this paper.

\section{References}

[1] Akiba, Y., Nakamura, K., Nishikawa, T. and Watanabe, T. (2012) History of Iyaku Bungyo. Yakuji-Nippo Co. Ltd., Tokyo.

[2] Ministry of Health, Labour and Welfare (2015). https://www.mhlw.go.jp/file/06-Seisakujouhou-11120000-Iyakushokuhinkyoku/hon bun_3.pdf

[3] Higuchi, K. (2014) Quantitative Text Analysis for Social Research. Nakanishiya Publication Co. Ltd., Kyoto.

[4] Kanda, Y. (2013) Investigation of the Freely Available Easy-to-Use Software "EZR" for Medical Statistics. Bone Marrow Transplant, 48, 452-458. https://doi.org/10.1038/bmt.2012.244

[5] Ministry of Health, Labour and Welfare (2018). https://www.mhlw.go.jp/file/06-Seisakujouhou-11120000-Iyakushokuhinkyoku/H2 9tyousahoukokusyo.pdf

[6] Ministry of Health, Labour and Welfare (2019). https://www.mhlw.go.jp/content/000509233.pdf

[7] Ministry of Health, Labour and Welfare (2016). https://www.mhlw.go.jp/file/06-Seisakujouhou-11120000-Iyakushokuhinkyoku/000 0112481.pdf

[8] Hirota, N., Inagaki, M., Matsumura, N. and Utsunomiya, R. (2018) An Outcome Evaluation of the Health Support Pharmacy Efforts to Improve Health Literacy. Japanese Journal of Pharmaceutical Health Care and Sciences, 44, 244-250. https://doi.org/10.5649/jjphcs.44.244 